\title{
Reflexiones sobre la representación de los grupos de poder urbanos y sus acciones en las crónicas medievales peninsulares*
}

\author{
Rethinking the representation of urban power groups and their actions within \\ the Iberian Medieval chronicles
}

Francisco José Díaz Marcilla

fdiaz@fcsh.unl.pt

Instituto de Estudos Medievais, Universidade Nova de Lisboa, Portugal

Recepción: 05 Agosto 2020

Aprobación: 03 Diciembre 2020

Publicación: 04 Enero 2021

Cita sugerida: Díaz Marcilla, F. J. (2021).

Reflexiones sobre la representación de los grupos de poder urbanos y sus acciones en las crónicas medievales peninsulares. Trabajos y Comunicaciones, (53), e141. https://doi.org/10.24215/23468971e141

\begin{abstract}
Resumen: El presente trabajo pretende abordar la representación que se hace en las crónicas de la Península Ibérica durante la Baja Edad Media de los grupos de poder de las ciudades, así como del pueblo llano, también como grupo de presión, en los reinos de Portugal, Castilla, Navarra, la Corona de Aragón y Granada. Se tratará de analizar las funciones que desempeñan esos grupos en las crónicas, destacando algunos ejemplos ilustrativos de cada reino.
\end{abstract}

Palabras clave: Crónicas medievales ibéricas, Baja edad media, Ciudades, Pueblo llano.

\begin{abstract}
This article aims to approach the representation of urban power groups within the Iberian Medieval chronicles in the Late Middle Ages, as well as the common, as pressure group. The kingdoms of Portugal, Castile, Navarre, the Crown of Aragon and Granada will be analysed, looking at which functions are carrying out by their urban powers. Some examples from each kingdom will be highlighted.
\end{abstract}

Keywords: Medieval Iberian Chronicles, Late Middle Ages, Towns, Common people.

\section{LAS FUNCIONES DE LOS GRUPOS URBANOS EN LAS CRÓNICAS}

A pesar de lo que se podría pensar a priori, el tema objeto de estudio en este trabajo no ha atraído la atención esperada por parte de la comunidad científica. Obviamente, existen numerosos estudios sobre la ciudad, su gobierno y las crónicas urbanas en todo tipo de casos y épocas, ${ }^{1}$ pero existen muchos menos sobre el aspecto que les propongo hoy aquí: la representación - entendida como la imagen transmitida en la narración - del gobierno de la ciudad y los grupos urbanos en el relato cronístico "oficial”, es decir, el relato avalado por el poder regio como narración oficial de los hechos históricos. ${ }^{2}$ Una historiografía medieval, además, que no distingue siempre claramente entre la "çibdad" general y las villas bajo jurisdicción específica de un rey (realengo), un noble (señorial), un abad (abadengo) o cualquier otro poder territorial.

Esta situación de tema casi inédito en los estudios cronísticos y/o históricos es comprensible si se tiene en cuenta que el gobierno de las ciudades medievales no es tampoco objeto de un interés particular por parte de los cronistas oficiales de los cinco reinos que se reparten el territorio de la Península Ibérica durante los siglos 
XIV y XV, a saber, Portugal, Castilla, Navarra, Aragón y Granada. Sin embargo, como han demostrado otros enfoques sectoriales similares, como la representación de la Iglesia y la religiosidad (Díaz Marcilla, 2018) o los "letrados" (Simões, 2018) en las crónicas bajomedievales, el interés se encuentra en los pequeños detalles que emergen de las crónicas y, a veces, aquello que las crónicas precisamente no dicen.

Cabe incidir en una idea ya formulada hace un tiempo (Nieto Soria, 2007) sobre la vocación propagandística de ese tipo de narrativa, como es la cronística, donde lo que realmente importa es el mensaje que se transmite, no su contenido verificable empíricamente. Siendo la monarquía y su ambiente de poder el que autoriza el relato, se explica esa intencionalidad de anular o, por lo menos, disminuir, la acción de otros poderes como puedan ser la Iglesia o las ciudades. De ahí, esa actitud del cronista de cierta desatención al fenómeno urbano y de solo sacarlo a colación si puede reforzar el discurso propagandístico que pretende transmitir.

Las crónicas oficiales distinguen fundamentalmente entre dos tipos de grupos en el cuerpo urbano: los "omes buenos/ homens bons/ homes bons/ señores/ qadíes" (Carlé, 1964) y el "pueblo/ povo/ poble/ común/ medina” (Asenjo González, 2004). Por tanto, el primer aspecto que salta a la vista es el maniqueísmo que los cronistas de cualquiera de los reinos realizan sobre el mundo urbano, pues en el primer grupo estarían incluidos tanto nobles como no nobles. Para el caso de las crónicas de ámbito musulmán, esa distinción queda todavía más diluida, pues el gobierno de la ciudad recae en manos de un estrechísimo grupo de familiares del rey o del emir. Por otro lado, el "pueblo" es percibido como una especie de entidad unitaria, que le servirá al cronista para protagonizar algunas acciones o secundar otras, como habrá ocasión de explicar más adelante.

En otro orden de cosas, como ya se indicó, se sabe por la documentación que las villas y ciudades podían ser de realengo, abadengo, dominio señorial o episcopal, pero esta distinción está muy diluida en las crónicas. Se suele especificar solamente si una villa o lugar pertenece al rey o a un noble - o muy raramente a un prelado o abad -, pero lo normal es que no se comente la vinculación político-administrativa, como ocurre con las crónicas aragonesas donde la autonomía de la ciudad prima sobre el resto de consideraciones. $\mathrm{O}$ el caso opuesto de las crónicas musulmanas, donde el gobierno de la ciudad es un apéndice del gobierno real, prácticamente sin autonomía.

Esta imagen pretende presentar al rey como "señor" de todo el territorio, y si hay alguna resistencia o problema en una villa, la causa se le atribuye siempre a algún noble rebelde. Especialmente ilustrativo es el caso portugués: según las crónicas de Fernão Lopes, durante el período de la Crise de 1383-1385, las ciudades y villas estuvieron siempre de parte del Mestre de Avis, y, en los casos en que la villa está de parte del rey castellano Juan I y su esposa, la infante heredera Beatriz de Portugal, en realidad se especifica que son los alcaides del castillo los que tienen voz por él, no el conjunto de los ciudadanos (Moreno, 1985).

\section{ACTORES: LOS "HOMBRES BUENOS"}

Trataremos ahora de dilucidar quiénes son estos "hombres buenos” o gobernantes de la ciudad que sí aparecen constantemente en la narrativa historiográfica oficial, cuya composición no siempre es clara, pues dentro de esta categoría se incluyen, por un lado, a los alcaides, regidores, jueces, notarios, contadores y oficiales, cuya designación suele ser regia; por otro lado, a los procuradores y a los jurados, cuya designación es por la propia ciudad o villa; y en un tercer segmento están, aunque no siempre, los mercaderes y burgueses adinerados (especialmente en las crónicas aragonesas). A veces están incluidos en la categoría de "hombres buenos", cargos específicos que solamente se dan en determinados lugares, como en Sevilla y Córdoba, los caballeros veinticuatro. Por el lado musulmán, nos informa Ibn Abi Zar' (1964, p. 416) que aclamaron a Yusuf I como nuevo califa de los almohades en 1176 los siguientes cargos de las ciudades de Ifriqiya, al-Magrib y al-Andalus: alfaquíes, qadíes, santones y jeques. Como se indicó antes, las fuentes musulmanas no hacen una denominación expresa de "hombres buenos", sino que mencionan a aquellos cargos o personas que destacan sobre los demás. 
La acción política de estos actores evidenciada en las crónicas deriva siempre de dos actitudes que les atribuye el cronista: la acción de "aprobación", si se quiere respaldar una acción del monarca (o de algún miembro de la familia real); o bien, si se quiere lo contrario, la acción "correctora” de alguna situación creada por el entorno real.

\subsection{La función de aprobación}

Relativamente a la primera actitud, existirían, a su vez, dos modalidades: la aprobación informal y la formal. La primera estaría caracterizada por la participación, en la mayoría de las ocasiones, festiva, de los buenos ciudadanos en algún acto celebrativo. Resulta excesivamente alto el número de ejemplos referidos en todas las crónicas peninsulares como para citarlos aquí, aunque resumidamente se pueden agrupar en tres tipos: las aclamaciones de un nuevo rey o el nacimiento del heredero; las concentraciones relacionadas con algún acontecimiento bélico, bien una victoria, bien la preparación de una batalla; y, sobre todo, con motivo del casamiento de alguien de la realeza. Característica común en todos estos casos es que acompañan siempre a los hombres buenos el resto de vecinos de la ciudad, o sea, el pueblo, actuando como una unidad en la aprobación informal de la acción regia.

Sin embargo, en el caso de la aprobación formal, el pueblo normalmente desaparece de la escena o es relegado a elemento que mira el transcurrir de la escena desde un segundo plano. De hecho, esta modalidad está reservada para momentos especialmente relevantes de la narración, y donde la participación de los poderes concejiles otorga un mayor peso propagandístico a la decisión tomada por la monarquía. Esta modalidad presenta una única forma de representación: la participación de los hombres buenos en las Cortes. Sabemos por la documentación de archivo que, en la mayoría de las ocasiones, los procuradores en las Cortes eran miembros de las élites urbanas o, más significativamente, de las élites del reino (Martínez Carrillo, 1990). Sin embargo, desde el punto de vista de la comunicación política, las crónicas prácticamente nunca refieren esta particularidad. En otras palabras: los procuradores son presentados únicamente como representantes de las ciudades, sin vínculos con el resto de esferas de poder. Normalmente, el nombre de los procuradores casi nunca es referido, excepto en situaciones muy específicas en las cuales se pretende rendir una especie de tributo al procurador que ha ayudado a la Corona. Por lo tanto, la representación de las ciudades, como un bloque unitario que apoya institucionalmente a la Corona, es utilizado por los cronistas como argumento cualitativo.

Pero hay algunos casos especiales, como el de Navarra. Según se sabe por la crónica de Garci López de Roncesvalles (1977, pp. 108-114), además de la "obligación" de jurar los fueros y libertades del reino de los reyes de Navarra, dicho juramento era correspondido y respondido por los procuradores, de tal manera que tras reproducirse el juramento del rey, se reproduce el juramento «de goardar bient et fielment la persona de nuestro seynnor el rey et de ayudar a goardar et deffender el regno a nuestro poder segunt nuestros fueros, husos, costumbres, privilegios, franquezas et libertades que cada uno de nos habemos». Por lo tanto, la crónica reutiliza el texto de la documentación - se conservan copias de los juramentos - evidenciando la paridad entre monarca y ciudades, lo que no es extraño ya que Navarra fue efectivamente el único reino cristiano donde la elección de reyes por parte de las Cortes no fue inusual (García Ramírez en el siglo XII, Teobaldo I en el siglo XIII, Juana II y Felipe de Évreux en el siglo XIV).

$\mathrm{Al}$ otro lado de la frontera religiosa, las crónicas oficiales musulmanas suelen referirse a la acción que desempeña un califa o emir cuando llega a una ciudad con la expresión "poner en orden sus negocios", lo que denota y refuerza esa dependencia del gobierno de las ciudades respecto al poder central. De hecho, tenemos al emir mandando construir mezquitas, escuelas, zocos, murallas, puentes, en los diferentes territorios del reino nazarí. Esta situación se ve reforzada si echamos un vistazo a las crónicas granadinas - de la mano de Ibn al-Jatib (2011) -, donde la ciudad pasa claramente a ser identificada con una unidad de producción de materias primas y de tributos. Los sabib de las ciudades como Almuñécar, Algeciras o Guadix, son miembros 
de la extensa familia real, pero no son cargos hereditarios, lo que muestra esa concepción del reino como un todo bajo el poder del sultán. Por último, nos hacemos eco del comentario de Ibn Jaldún en su Introducción a la Historia Universal(1977, pp. 287-288) en que explica que las personas importantes de las ciudades, para conservar su riqueza, se deben poner bajo la protección de un miembro de la familia real. Es decir, no hay un verdadero poder identificado en las crónicas.

\subsection{La función correctora}

Por el otro lado, la acción "correctora" muestra de una manera clara y evidente cómo se era consciente del poder que podían llegar a tener las ciudades en cuanto colectivos sociopolíticos. Más concretamente, sus dirigentes. Esta podría ser la razón para que aparezcan en el relato cronístico: consiguen modificar la acción regia porque es bueno para el reino. Cabe subrayar que, para este tipo de acciones, no existe otra modalidad que la formal, dada la relevancia que tiene a nivel político y simbólico en la propaganda (es difícil narrativamente contradecir al rey, si no es a través de mecanismos formales).

Sirva como primer ejemplo el peso político de los representantes - los procuradores - de las ciudades, en momentos como en la minoría de Enrique III de Castilla entre 1390 y 1393 . En esa ocasión nos cuenta Pedro López de Ayala (1780, pp. 414-416) cómo se decidió, después de largas discusiones entre los nobles - que duran por lo menos 15 capítulos de su Crónica del rey Enrique III -, que estuvieran en el consejo de regencia los procuradores de las 6 ciudades más importantes del reino: Burgos, León, Toledo, Sevilla, Córdoba y Murcia. Ayala sugiere, y esto es lo relevante, que este conflicto se zanjó en 1392 gracias a la intervención soterrada de los procuradores de los concejos, que hicieron presión para que al final se ejecutase la voluntad del testamento de Juan I. Además, el cronista especifica que los que tienen que elegir a los candidatos en cada ciudad son «el concejo e oficiales e omes buenos» de la misma. Otro ejemplo de autonomía en las decisiones en la Corona de Castilla está en la Crónica del Halconero, de Pedro Carrillo de Huete (1946, p. 353), cuando las villas de Cáceres y Trujillo se negaron a darse al conde Pedro de Estúñiga en 1440, a pesar de que el rey lo requiriese así. En este caso el cronista alaba esta decisión porque las villas "sabían" que el rey en realidad no quería eso pues eran villas de realengo.

Otro ejemplo de esta segunda acción nos lo ofrece la Chrónica de el-Rei D. Fernando, de Fernão Lopes donde se observa claramente esa implicación de los notables de las ciudades, u "homens bons", en la acción de corregir, pues se dice que instaron a la regente Leonor Teles, tras la muerte de Fernando I de Portugal en octubre de 1383, a que escogiera para el gobierno del reino a varias figuras, y, entre ellas, dos «homens bons cidadãos e entendidos», representantes de cada una de las comarcas de Portugal (Lopes, 1896, 3, pp. 182-187).

No es muy común, pero puede ocurrir a veces que el poder concejil represente una amenaza para el poder regio. Así, la Crónica de Pere el Cereminiós, referida a Pere IV de Aragón, narra los problemas que tuvo el rey con la ciudad de Valencia entre 1347-1349, cuando la revuelta de la denominada Unió, donde nobles rebeldes se aliaron con ciudadanos descontentos con las políticas regias y obligaron al monarca a otorgar libertades no pretendidas por él. Pasados los dos años que duró el movimiento, el rey aprovechó la debilidad de la Unió para encarcelar a los cabecillas de la revuelta y, tras un rápido juicio sumarísimo, colgar de las murallas de Valencia los cuerpos de aquellos que osaron enfrentarse con él (Ceremonioso, 1850, pp. 244-249).

Volviendo sobre las Cortes, si ayudan en la acción aprobadora formal, desempeñan igualmente una importante acción "correctora". A pesar de toda la exaltación de reyes y de la familia real, los cronistas oficiales no consiguen eludir el peso de las Cortes a la hora de objetivar el verdadero papel de los monarcas. En otras palabras, estos poco pueden hacer sin el parecer positivo de las Cortes. Normalmente, las crónicas reflejan el momento de la reunión de Cortes, pero, siempre que pueden, lo obvian o lo reducen a un simple trámite. Que esto se corresponda con la realidad es discutible. Por otro lado, en momentos de especial crisis, tenemos que las reuniones de Cortes cobran un papel protagonista cuya dimensión dependerá del apego que el cronista 
tenga al monarca en cuestión. Uno de los ejemplos más evidentes es el de Ayala, en su Crónica del rey Juan I, cuando narra lo que ocurrió en las Cortes de Guadalajara de 1390: los procuradores no solo se negaron a pagar dineros extra al rey, sino que le exigieron controlar mejor el número de caballeros pagados por la Corona, pues eran demasiados y las arcas reales estaban malgastándose en mercedes sin control (López de Ayala, 1780, pp. 311-314). En el texto cronístico destacan los procuradores de las ciudades de Toledo, Sevilla, Burgos y León, como principales interlocutores de la Corona. Al final, las ciudades pagaron, pero también los nobles, lo que puede ser interpretado - aunque Ayala no opine al respecto - como una victoria de los ciudadanos.

En la Crónica del Halconero, se ve a Juan II teniendo que posponer su acción guerrera contra los musulmanes, así como de pacificación de la nobleza rebelde porque las Cortes, alegando que faltaban procuradores, no podían empezar, ni, consecuentemente, aprobar el pedido de dinero del monarca (Carrillo de Huete, 1946, p. 466). Hay otro pasaje muy ilustrativo referido al Reino de Aragón en el que los procuradores de las ciudades rehúsan pagar un dinero para sostener la guerra que el rey de Navarra e infante de Aragón, D. Juan, y otros nobles rebeldes quieren hacerle al rey de Castilla y, más especialmente, al condestable Álvaro de Luna. Así que estos tuvieron que buscar la financiación por otros lados, acudiendo a «otros fauores de cavalleros e presonas singulares» (Carrillo de Huete, 1946, p. 528).

Sin embargo, el momento culminante en este sentido lo tiene la Chrónica de el-Rei D. João de Fernão Lopes, en la que son las Cortes quienes deciden que, no habiendo un heredero legítimo - la crónica es pródiga en detalles a la hora de hablar de las ilegitimidades de los diferentes aspirantes al trono -, debe ser el "reino", entendido como el conjunto de representantes del mismo, y entre ellos, las ciudades, quien elija a su nuevo rey (Lopes, 1897-1898, 3, pp. 174-219). Es un caso paradigmático pues se sabe que, en realidad, no estuvieron todos los procuradores de todas las ciudades, ni todos los nobles, ni todos los eclesiásticos.

\section{ACTORES: EL “COMÚN”}

El segundo de los grupos sociales más relevantes de las crónicas peninsulares medievales es el denominado como "povo" o "pueblo" o "poble" o "comn". No tiene, efectivamente, ningn tipo de control sobre la ciudad ni es representante activo de la misma en cuanto sujeto político. Sin embargo, los cronistas son conscientes que muchas de las acciones propuestas por los "hombres buenos", si no tienen la aquiescencia de las clases populares, poco o nada tienen que hacer. Por ello, se reproduce el doble modelo de "aprobación" o de "corrección" comentado anteriormente.

\subsection{La acción de aprobación}

En el primer caso, suele ser utilizado como elemento activo o pasivo de un episodio histórico, pero, aun así, casi nunca se trata de algo espontáneo, sino resultado de una acción orquestada por algún miembro de la élite. Por clarificar, tenemos los ejemplos de la Crónica de Pere el Cerimoniós, en la que el pueblo de Perpiñán es congregado en 1344, el de Barcelona en 1354 y el de Murviedro en 1364 para que asistan a los pregones que hace el propio rey Pere IV (Ceremonioso, 1850, pp. 218-219, 314 y 362). No hay una participación activa en este caso, pero sí una aprobación pasiva de la acción del monarca.

Por otro lado, está el ejemplo mixto del pueblo de Lisboa ayudando activamente, con armas, y pasivamente, con rezos y procesiones, al Mestre de Avis durante el cerco de la ciudad por las tropas del contingente lusocastellano en 1384. De su poder como elemento decisivo del panorama político nos da fe Fernão Lopes (1897-1898, 1, pp. 84-89) cuando habla en su Chrónica de el-Rei D. João del «commum povo livre» de Lisboa, y, tras la reunión del Mestre de Avis con los ciudadanos en el Monasterio de São Domingos en diciembre de 1383, la incorporación de 24 hombres en representación de los oficios de la ciudad (dos por cada uno) como miembros del Consejo de Regencia. 


\subsection{La acción correctora}

Como ejemplo de una acción correctora del pueblo llano, tenemos el caso del pueblo de Torrijo, que era de la Corona de Aragón, donde Pedro I puso a un alcalde tras tomar la villa en 1358, pero que el pueblo mató en desacuerdo (López de Ayala, 1779, p. 252). También tenemos a los «mesteiraes de todos mesteres e besteiros e homens de pe» de Lisboa, yendo al palacio de Fernando I para quejarse por la sorpresiva boda de éste con Leonor Teles en 1372. Precisamente por no darles respuestas es, según la crónica, el motivo por el que el "común" se pondría de parte del Mestre de Avis (Lopes, 1895-1896, 1, p. 192).

Para las crónicas musulmanas, en Rawd al-Qirtas encontramos una definición bastante elocuente de lo que es el "común" para el cronista, al hablar sobre una sublevación a la que se apuntaron «los cristianos y alguna gente baja de los que no conocen las consecuencias de las cosas» $(1964$, p. 726). Una opinión parecida se intuye en la Historia de los reyes de la Alhambra de Ibn al-Jatib, quien habla de un episodio en que la plebe, al enterarse de la muerte del visir de Muhammad III en 1309 durante su destronamiento, entró al pillaje en las casas del visir, lo que califica como «una gran pérdida para los musulmanes, porque se dispersaron en manos ruines» (2011, p. 67). En otra ocasión, en 1314, durante los disturbios motivados por la lucha entre las tropas de Nasr I e Ismail I, comenta este cronista con desprecio que «se apresuró el vulgo y la hez del pueblo [...] a escalar los alminares y los lugares altos de recreo y las colinas» (2011, p. 87).

\section{OTROS FACTORES: LA GUERRA}

Conviene hacer una pequeña digresión al respecto de la ciudad y su papel en el contexto bélico bajomedieval, tal como descrito por las crónicas oficiales. No siendo el objetivo de este trabajo, tampoco se examinarán todos los casos, aunque sí será un complemento importante para la finalidad de este trabajo. ${ }^{3}$

Uno de los símbolos de poder de las ciudades es visible en las crónicas únicamente en acontecimientos bélicos. Concretamente, es en la frontera donde más aparece este símbolo, como signo de la adhesión del reino al monarca: el pendón de guerra. De manera constante, los pendones de Sevilla, Córdoba, Jerez o Jaén serán vistos con un buen contingente de tropas procedentes del cuerpo urbano para atacar a los musulmanes o en el transcurso de una acción defensiva (Millán Crespo, 1987; Segura, 2007). Por el lado andalusí - e islámico en general -, hay pendones de guerra, pero más asociados a un miembro de un linaje que no a la ciudad de donde sea el sahib('Athamina, 1989).

En el caso de Ayala (1780, pp. 216 y 241-242) y Fernão Lopes (1897-1898, 6, pp. 51-52), la guerra lusocastellana servirá para ensalzar la lealtad de determinadas villas, así como la potencia guerrera de los reyes, dependiendo de si la villa es tomada o es abandonada a su suerte. En este contexto, hay que destacar el hecho de que sistemáticamente Juan I va diciendo a una tras otra de las villas y lugares que aún tenían su voz en Portugal, que sus cuidadores las entregaran a los portugueses (Chaves, Bragança, Guimarães, Campo Maior). En otros casos, no es el rey sino algunos nobles y ciudadanos los que deciden defenderla, caso de Mértola. Otros, son directamente abandonados por sus custodios (caso de Alenquer, Óbidos o Sintra).

Dentro del contexto de la guerra, aunque interna en este caso, hay otro aspecto de especial relevancia para nuestro análisis del poder urbano, o mejor, de los poderes urbanos: conocemos por las fuentes documentales la existencia de luchas internas en las ciudades, calificadas como luchas de bandos, "banderizos", etc. Lo curioso es que, para la mayor parte de los cronistas oficiales, este tema queda omitido, o totalmente relegado a un plano secundario. Para el caso castellano, las luchas en Sevilla entre los Ponce de León y los Guzmanes (Sánchez Saus, 1991), o en Murcia entre los Fajardo y los Manueles (Martínez Carrillo, 1985) siempre se analiza y es presentado como luchas entre partidarios del rey y rebeldes antimonárquicos. Para el caso portugués, es Fernão Lopes quien presenta las luchas dentro de las ciudades y villas durante la Crise de 1383-1385 como luchas de los «verdadeiros portuguezes» contra los «falsos portuguezes» (Lopes, 1896, 3, p. 131). O 
sea, se altera el sentido de las luchas urbanas, encuadrándolas en el contexto de Portugal contra el enemigo castellano.

A este respecto, para 1434, tenemos el testimonio de la Crónica del Halconero(Carrillo de Huete, 1946, p. 531) y de la Refundición del Halconero de Lope de Barrientos (1946, p. 155), que anticipan una de las figuras más importantes de la política urbana del siglo XV en Castilla: el corregidor (Bermúdez Aznar, 1974). Su aparición está ligada en estas crónicas a la necesidad, por parte de las propias ciudades, de garantizar la paz dentro de las murallas, tratando de eliminar o disminuir el impacto de las "luchas de bandos". Son los propios concejos los que piden que haya un elemento externo de mediación que dependa totalmente del monarca. Es, por tanto, el monarca en última instancia quien ofrecerá una solución duradera para este problema, a petición de las ciudades.

\section{Otros FACTOREs: LA Iglesia}

Cabe también resaltar un aspecto que atañe a otro de los componentes del entramado social urbano y que caracterizan también su vida política. Me voy a referir al clero urbano, bien perteneciente a una catedral, bien a alguno de los monasterios y conventos ubicados dentro o en las cercanías de los núcleos urbanos. De ellos, hay numerosos testimonios de su labor y relevancia política en la documentación administrativa, teniendo un destacado papel en ocasiones delicadas, como en guerras civiles o en vicisitudes de carácter político. Sin embargo, esa relevancia es desdibujada en el relato cronístico, especialmente en lo que al clero secular se refiere, encontrándolo reducido a un mero papel circunstancial en las crónicas de prácticamente todos los reinos cristianos de la península (Díaz Marcilla, 2018). En efecto, si aparecen obispos o arzobispos, la mayoría de las veces es sin ser mencionados por nombre y sin estar ligados a una ciudad, siendo debida su mención al hecho de estar o no en el séquito de un rey, una reina o un infante. Su impacto en la historia urbana queda reducido a casi una anécdota.

En una concepción diferente están los miembros del clero regular, cuya acción sí es en varias ocasiones crucial para la narración historiográfica. De manera destacada están representados en las Chrónica de el-Rei D. Fernando y de el-Rei D. João I, pues, con el trasfondo del Cisma de Occidente, la acción de los religiosos se convierte en una cuestión entre católicos y cismáticos. Sucederá que los frailes franciscanos y benedictinos servirán de mediadores entre las fuerzas en conflicto en Oporto, Estremoz, Guimarães y Ponte de Lima (Lopes, 1897-1898, 1, pp. 132; 2, p. 145; 4, pp. 57 y 67); y sucederá con frailes franciscanos, como Rodrigo de Cintra o Pedro de Lisboa, que hacen sermones de enaltecimiento del nuevo rey portugués ante el pueblo de Lisboa (Lopes, 1897-1898, 3, pp. 66-72; 4, pp. 192-200).

\section{Conclusiones}

Tras el análisis realizado hasta aquí, cabe establecer una serie de conclusiones, provisionales algunas, más cimentadas otras, sobre la presencia y modo en que las élites urbanas y el pueblo en general son representados y usados por los cronistas oficiales de los reinos cristianos y musulmanes para reforzar la narración de la historia de cada reino.

Así pues, en primer lugar, se puede concluir que en todas las crónicas peninsulares hay una doble función asociada a las élites urbanas y al pueblo llano como "aprobadores" o "correctores" de las políticas de la monarquía y de los miembros de la familia real. Esta visión se enmarca en la pretensión última de los cronistas a la hora de construir sus relatos, que consiste, en definitiva, en utilizar al mundo urbano como un elemento más, encaminado a enaltecer la figura del rey y del regimiento monárquico.

En segundo lugar, si se compara esta imagen con la que muestran los documentos de cancillería real o de cancillerías urbanas conservados, queda claro que la cronística no deja entrever deliberadamente el verdadero 
papel que jugaron las ciudades en el entramado histórico de los reinos peninsulares medievales. El motivo es claramente político: no hay más figura poderosa que el rey y la monarquía, siendo todo lo demás elementos complementarios, pero no sustanciales, de la "historia oficial". Incluso en los casos, como las Cortes de Coímbra en Portugal, donde el cronista no puede eludir el peso de las ciudades en el éxito del cambio de línea sucesoria, la escena es presentada como la ineludible necesidad de hacer rey al que ya actuaba como tal, el Mestre de la Orden de Avis.

En tercer lugar, ya en el ámbito de la conjetura, hay un aspecto simplemente esbozado en este estudio, sobre el que valdría la pena profundizar. Se trata de una cuestión semántica, que se observa en la mayoría de las crónicas: cuando se está hablando de determinados momentos y episodios proclives a la monarquía, los cronistas llaman al pueblo que aclama al rey "ciudadanos" y/o "vecinos"; en cambio, cuando los momentos descritos son adversos y tumultuosos, la denominación utilizada para la gente congregada es "muchedumbre" o "pueblo" seguido de algún adjetivo despreciativo. Esta personificación de grupos sociales encaja perfectamente con la mencionada intencionalidad del cronista, aunque al mismo tiempo abre una reflexión, todavía por hacer, sobre la relación monarquía-reino y la manera en que se va desarrollando a lo largo de los siglos bajomedievales para desembocar en el conocido como estado moderno.

Por último, cabe indicar otra vía de posible estudio sobre este tema, consistente en la comparación de esta realidad aquí comentada con otras situaciones en otros contextos. Me refiero, más concretamente, a cómo la narrativa historiográfica de Francia, Inglaterra, el Sacro Imperio o las comunidades italianas abordan esa relación entre poder central y poder periférico a nivel de relato, analizando las justificaciones o comentarios que hacen sobre ella en contextos tan conflictivos como la Guerra de los Cien Años.

\section{ReFERENCIAS}

Abi Zar', Ibn (1964). Rawd al-Qirtas. Antonio Huici Miranda (ed.). Valencia: Anubar.

Al-Jatib, Ibn (2011). Historia de los reyes de la Alhambra. Emilio Molina López (ed.). Granada: Universidad de Granada.

Asenjo González, María (2004). El pueblo urbano: el «común». Medievalismo. Revista de la Sociedad Española de Estudios Medievales, (13-14), 181-194.

Asenjo González, María (2005). Las ciudades medievales castellanas: balance y perspectivas de su desarrollo historiográfico (1990-2004). En la España Medieval, (28), 415-453.

Asenjo González, María (2013). Preparar la paz y prevenir la guerra en las ciudades medievales. En Ana Arranz, María del Pilar Rábade y Óscar Villarroel (coords.), Guerra y paz en la Edad Media (pp. 109-140). Madrid: Sílex.

'Athamina, Khalil (1989). The Black Banners and the Socio-Political Significance of Flags and Slogans in Medieval Islam. Arabica, (36), 307-326.

Barrientos, Lope de (1946). Refundición de la Crónica del Halconero. Juan de Mata Carriazo (ed.). Madrid: Espasa Calpe.

Bermúdez Aznar, Agustín (1974). El Corregidor en Castilla durante la Baja Edad Media (1348-1474). Murcia: Universidad de Murcia.

Carlé, María del Carmen (1964). "Boni homines" y hombres buenos. Buenos Aires: Facultad de Filosofía y Letras de la Universidad de Buenos Aires.

Carrillo de Huete, Pedro (1946). Crónica del Halconero de Juan II de Castilla. J. de Mata Carriazo (ed.). Madrid: Espasa Calpe.

Ceremonioso, Pedro IV (1850). Crónica de Pere el Cerimoniós. Antoni de Bofarull (ed.). Barcelona: Imprenta de Alberto Frexas.

Díaz Marcilla, Francisco José (2018). El período aviñonés y el Cisma como trasfondo de los conflictos internos en la cristiandad: una perspectiva desde la narrativa historiográfica (1309-1417). En Luis Araus y Juan Antonio 
Prieto (coords.), Las tres religiones en la Baja Edad Media peninsular. Espacios, percepciones y manifestaciones (pp. 187-202). Madrid: La Ergástula.

Esteve Secall, Rafael (2000). Notas sobre una teoría de la ciudad en Ibn Jaldún. Cuadernos de Ciencias Económicas y Empresariales, (38), 169-193.

Jaldún, Ibn (1977). Introducción a la historia universal. Al Muqaddimah. México: Fondo de Cultura Económica.

Lopes, Fernão (1896). Chrónica de el-Rei D. Fernando, vol. 3. Lisboa: Escriptório.

Lopes, Fernão (1897-1898). Chrónica de el-Rei D. João I, 7 vols. Lisboa: Escriptório.

López de Ayala, Pedro (1779). Crónicas de los Reyes de Castilla: Pedro I, vol. 1. Eugenio de Llaguno Amirola (ed.). Madrid: Imprenta de D. Antonio de Sancha.

López de Ayala, Pedro (1780). Crónicas de los Reyes de Castilla: Enrique II, Juan I y Enrique III, vol. 2. Eugenio de Llaguno Amirola (ed.). Madrid: Imprenta de D. Antonio de Sancha.

López de Roncesvalles, Garci (1977). Crónica de los Reyes de Navarra. Carmen Orcástegui Gros (ed.). Pamplona: Universidad de Navarra.

López Pérez, Miguel Ángel (2007). La ciudad en la historiografía bajomedieval y altomoderna hispana en los últimos treinta años: líneas para el estudio de la cultura e identidad urbana. Miscelánea Medieval Murciana, (31), $111-122$.

Martínez Carrillo, María de los Llanos (1985). Manueles y Fajardos: la crisis bajomedieval en Murcia. Murcia: Caja de Ahorros de Murcia.

Martínez Carrillo, María de los Llanos (1990). Sobre los mecanismos de extracción de los procuradores a Cortes en la Baja Edad Media: el caso de Murcia. En Las Cortes de Castilla y León, 1188-1988 (pp. 341-352). León: Cortes de Castilla y León.

Millán Crespo, Juan Antonio (1987). Estandartes medievales hispanos a través de la fuentes iconográficas y escritas. En II Congreso de Arqueología Medieval Española (Madrid, 19-24 enero 1987), vol. III (pp. 13-21). Madrid: Dirección General de Cultura, Comunidad de Madrid-Asociación Española de Arqueología Medieval.

Monsalvo Antón, José María (2010). La imagen de las ciudades y regiones altomedievales de León y de Castilla en las Crónicas Generales (de Sampiro a la «Estoria de España»). Studia Historica. Historia medieval, (28), 83-123.

Moreno, Humberto Baquero (1985). A campanha de D. João I contra as fortalezas da região de Entre-Douro-e-Minho. Revista da Faculdade de Letras. Historia, (2), 45-58.

Nieto Soria, José Manuel (2007). Propaganda y opinión pública en la historia. Valladolid: Secretariado de Publicaciones de la Universidad de Valladolid.

Sánchez Saus, Rafael (1991). Linajes sevillanos medievales, 2 vols. Sevilla: Guadalquivir.

Segura González, Wenceslao (2007). Los pendones de la batalla del Salado. Aljaranda: revista de estudios tarifeños, (66), 9-46.

Simões, José (2018). As representações do saber. Uma visão dos letrados nas crónicas portuguesas tardomedievais. Tese de Mestrado. Évora: Universidade de Évora.

\section{Notas}

* Este trabalho é financiado por fundos nacionais através da FCT - Fundação para a Ciência e a Tecnologia, I.P., no âmbito da Norma Transitória - DL 57/2016/CP1453/CT0103.

1 La lista es amplia, siendo destacables y útiles para una panorámica general: Asenjo González, 2005; López Pérez, 2007; Monsalvo Antón, 2010.

2 De hecho, existen solo análisis parciales sobre el ámbito musulmán en la obra de Ibn Jaldún (Esteve Secall, 2000) y alguna aproximación en el ámbito cristiano (Soler, 2011).

3 Invito a los interesados a leer el trabajo de María Asenjo González (2013) para una aproximación al tema. 